\title{
PROPUESTA MEDOTOLÓGICA DE RESTAURACIÓN ECOLÓGICA EN LOS PÁRAMOS DEL VOLCÁN CHILES, NARIÑO
}

\section{Methodological proposal of ecological restoration in paramos of the volcano Chiles, Nariño}

\author{
AIDA ELENA BACA G. ${ }^{1}$ \\ 1Universidad del Valle.
}

E-mail: aida.baca@correounivalle.edu.co

\section{Resumen}

A partir de conceptos como Teoría general de sistemas, sistemas complejos y ecología de la restauración, se propone una metodología para recuperar zonas degradadas por disturbios antrópicos en paramos. Para ello se describe el ecosistema, su problemática y las acciones que conducirán a determinar cuáles son los sitios, especies y condiciones de clima que favorecen en un proceso de restauración ecológica para que el ecosistema paramuno se regenere más rápidamente.

Palabras claves: Disturbios, Estabilidad, Índice, Páramos, Recuperación, Resilencia.

\begin{abstract}
Since concepts such as general systems theory complex systems and restoration ecology, proposes a methodology to recover degraded areas by anthropogenic disturbances in páramo. We describe the ecosystem, their problems and actions that will lead to identifying the sites, species and weather conditions that favor a process of ecological restoration for that the páramo ecosystem will can to regenerate faster
\end{abstract}

Keywords: Disturbance, Stability, Index, Paramos, Recovery, Resilience. 


\section{Restauración ecológica en los páramos}

\section{INTRODUCCIÓN}

La continua extracción de recursos naturales y la contaminación generada por los patrones de producción y consumo, están presionando los diferentes ecosistemas colombianos y por lo tanto, afectando la sostenibilidad ambiental. De acuerdo con la Política Nacional de Producción y Consumo sostenible (2009), si los recursos naturales se agotan no hay desarrollo y menos sustentabilidad, por lo tanto, es necesario cambiar los modelos de producción y consumo actuales, ya que son éstos los que extraen la materia prima de la naturaleza y emiten vertimientos, producen residuos y otros tipos de contaminación que impactan al ambiente.

En Colombia, desde 1997, a partir de la Política Nacional de Producción más limpia, ha existido una preocupación por prevenir, mitigar, corregir y compensar los impactos ambientales sobre la población y los ecosistemas. Por ello, a partir de ese año, se adquirió como un compromiso dentro del convenio marco de concertación para una producción más limpia, la recuperación de los ecosistemas.

Un ecosistema fuertemente presionado por diversas actividades humanas es el páramo. Por ello, teniendo en cuenta los impactos ambientales que ocurren hoy en día en las zonas paramunas, así como su importancia y vulnerabilidad; a partir de la conceptualización existente sobre Ecología de la Restauración, Teoría General de Sistemas, sistemas complejos, dinámica de ecosistemas, Disturbios y Restauración, se desarrollará una propuesta metodológica respecto a cómo valorar la respuesta de resiliencia de un ecosistema de páramo al aplicarle actividades de restauración ecológica.

\section{Páramo como ecosistema}

Según la teoría general de sistemas, para Bertalanffy (1976) y Van Gigch (1987), los sistemas abiertos son aquellos que presentan relaciones de intercambio con el ambiente, a través de entradas y salidas; intercambian energía, materia e información; son eminentemente adaptativos de acuerdo a las condiciones del medio para sobrevivir. Además, evitan el aumento de la entropía y pueden desarrollarse en dirección a un estado de creciente orden y organización (entropía negativa) y además restauran su propia energía.

Actualmente los sistemas ambientales son considerados sistemas complejos. El carácter de "complejo" está dado por las interrelaciones entre los componentes, cuyas funciones dentro del sistema no son independientes. El conjunto de sus relaciones constituye la estructura, que da al sistema la forma de organización que le hace funcionar como una totalidad. García (1994), afirma que los sistemas como totalidades organizadas tienen dos características fundamentales:

a) Las propiedades del sistema en un momento dado, no resultan de la simple adición de las propiedades de los componentes. La vulnerabilidad o resiliencia, así como las condiciones de estabilidad, son propiedades estructurales del sistema en su conjunto.

b) La evolución del sistema responde a una dinámica que difiere de las dinámicas propias de sus componentes, de tal manera que el sistema total integra, en su evolución, procesos de escalas temporales que varían considerablemente entre los subsistemas, e induce cambios en estos últimos.

De acuerdo a lo anterior, por su naturaleza, los páramos son considerados como un sistema abierto y complejo, con características especiales de: clima con una temperatura media anual que fluctúa entre $4^{\circ}$ y $10^{\circ} \mathrm{C}$, precipitaciones que varía según sea un páramo seco o húmedo desde 623 a 4000 $\mathrm{mm}$ anuales, humedad relativa promedio del $85 \%$; suelos ácidos originados a partir de piroclastos y cenizas volcánicas; altitud que va desde los 3000 hasta por encima de los 4100 metros; y altos valores de diversidad y endemismo tanto en fauna como en flora, con plantas adaptadas morfológica y fisiológicamente a condiciones climáticas y edáficas extremas.

Los ecosistemas paramunos ofrecen una gran variedad de servicios ambientales entre los que se destacan: continua provisión de agua en cantidad y calidad, de la que dependen los sistemas de riego de agua potable e hidroelectricidad; almacenamiento y fijación de carbono atmosférico que ayuda a controlar el calentamiento global; sistema productor que los ha constituido en centros de desarrollo cultural, étnico y campesino; y atractivos escénicos y científicos que proporcionan oportunidades para el turismo ecológico, la observación y la investigación científica (Hofstede et al. 2003).

En general, los páramos en toda la zona andina, están habitados por comunidades indígenas, quiénes desde su cosmovisión los consideran como áreas sagradas y en su plan de vida ambiental la conservación de estos ecosistemas es una actividad prioritaria. Desde el punto de vista sociocultural, estas áreas han jugado un papel relevante respecto de la relación hombre - montaña, pues es el espacio de donde obtienen alimento, medicina, materiales para la construcción de sus viviendas y leña, así como el lugar 
sagrado y de gran valor en mitos y leyendas.

Según el documento de Los pueblos indígenas y el medio ambiente (2010), las leyes, las costumbres y prácticas tradicionales reflejan tanto una adhesión a la tierra como la responsabilidad por la conservación de las tierras tradicionales para su uso por las generaciones futuras.

\section{Problemática ambiental en los páramos}

El páramo constituye un todo sinérgico, orientado hacia determinados propósitos y en permanente relación de interdependencia con el ambiente externo. Sin embargo, está siendo impactado por procesos sociales y culturales que determinan la causalidad de la problemática ambiental que en el ecosistema paramuno está ocurriendo.

En la actualidad, las comunidades tanto indígenas como campesinas que habitan los complejos paramunos, se dedican a la extracción de leña y madera, a la explotación de hielo y azufre, al monocultivo de papa y a la ganadería, así como a la extracción de recursos no maderables con distintos fines (Rivas et al. 2006). El desarrollo de estas acciones implica en algunos casos la realización de quemas continuas.

De acuerdo con Rangel (2000) y Vargas \& Mora (2007), toda esta continua y progresiva actividad social, ha transformado y modificado los páramos tanto en su estructura y función ecosistémica como en su extensión, uso, manejo y adecuación causando perturbaciones que producen cambios y descontrol en los suelos, vegetación, clima y oferta hídrica, alterando los regímenes de disturbios naturales y el equilibrio dinámico propio del ecosistema, generando un paisaje paramuno altamente heterogéneo a manera de mosaico con trayectorias sucesionales en parches y con diferentes edades y frecuencias de quema.

\section{Estabilidad del Ecosistema y Disturbios Antrópicos}

En general, en los páramos se cumple lo propuesto por Jørgensen \& Fath (2004) quienes consideran que "los procesos biológicos usan la energía captada para apartarse del equilibrio termodinámico manteniendo un estado de baja entropía respecto a su entorno. Después de la captura inicial de energía a través de la frontera, el crecimiento y desarrollo del ecosistema puede hacerse: incrementando la estructura física (biomasa), incrementando las redes (más ciclos) o incrementando la información incorporada al sistema. Las tres formas de crecimiento implican que el sistema se aleja del equilibrio termodinámico". Respecto al recurso suelo, Zúñiga et al. (2009), sostienen que la energía libre de Gibbs es mayor en un ecosistema sin intervenir que en uno intervenido con labranza intensiva, encontrándose que la Reserva Energética del Suelo o el Potencial Productivo del Suelo (PPS) es mayor en el ecosistema sin intervenir.

Igualmente, la estabilidad de los sistemas ecológicos está compuesta por: a) la resiliencia o rapidez con la que el sistema regresa a sus condiciones originales después de una perturbación y b) la resistencia o capacidad del sistema para soportar disturbios. Sin embargo, la capacidad que tiene un ecosistema para regresar a sus condiciones originales se hace evidente cuando un disturbio lo aleja de su estado basal (Wu \& Loucks 1995).

Para Wooton (1998), los disturbios de origen humano como deforestación para actividades ganaderas, agricultura de subsistencia en pequeñas superficies, pastoreo dentro del bosque y la extracción forestal selectiva fragmentan áreas continuas de ambientes naturales alterando la conectividad; de tal forma que normalmente no liberan recursos y modifican tan profundamente algunas propiedades del sistema que las especies no tienen la capacidad de aprovecharlos. En ocasiones el régimen de perturbación es tan fuerte que el ecosistema no puede asimilarlo, encontrándose sometido a un factor tensionante o de estrés que impide la capacidad de regeneración, lo cual trae como consecuencia la degradación, daño, transformación o total destrucción hasta tal grado que el ecosistema no se puede restablecer por su cuenta y retornar al estado anterior a la alteración o a su trayectoria histórica de desarrollo (Premauer \& Vargas 2004).

\section{Estrategia para recuperación un ecosistema perturbado}

Los disturbios pueden afectar la capacidad productiva de un ecosistema a través de cualquiera de las siguientes vías: cambiando los patrones espacio-temporales de disponibilidad y reciclaje de nutrientes, adicionando o removiendo biomasa, cambiando la tasa de sucesión vegetal (principalmente debido a consumidores que cambian las interacciones competitivas de las plantas) y cambiando la razón materia viva / materia muerta del sistema (Pickett \& White 1985, Hobbs \& Huennecke 1992).

Las respuestas de un ecosistema al disturbio dependen, obviamente, de las características de la alteración, pero también de las propiedades dinámicas de aquél.

Una estrategia para la reparación es la restauración ecológica; entendida como la actividad deliberada que inicia 
Baca (2011)

o acelera la recuperación de un ecosistema con respecto a su salud, integridad y sostenibilidad.

De acuerdo con diferentes autores como Jackson et al. (1995) y la Sociedad internacional para la restauración ecológica (SER 2004), la restauración ecológica es un proceso inducido por el hombre para recuperar las condiciones ambientales (flora, fauna, microorganismos, clima y suelo) de un ecosistema perturbado. En este proceso se trata no solo de rescatar especies sino recuperar las interacciones y procesos ecológicos donde las especies están relacionadas entre sí con el medio abiótico (Jiménez et al. 2002).

Cuando se realiza un proceso de restauración en un ecosistema, se emplean diferentes estrategias y prácticas tanto de las ciencias naturales como sociales, que conduzcan a restablecer de forma asistida las dinámicas naturales de éste. Sin embargo, los resultados son impredecibles, pues unos casos funcionan pero otros no, debido a que existen algunos factores responsables de la resiliencia de los sistemas degradados como: preferencia de especies; interacciones tróficas; conectividad de paisaje; fuentes de semillas; actividades sociales y culturales locales.

Es conveniente detectar cuáles de ellos son los que causan la resiliencia, para enfocar adecuadamente los trabajos de restauración. Si hay un sólo factor responsable, las probabilidades de controlar las transformaciones del ecosistema son altas, pero si hay varios es fundamental detectar cuál es el más importante.

De acuerdo con Suding et al. (2004), es interesante saber que para romper los ciclos que dan resiliencia a los ecosistemas degradados, puede ser necesario (casi paradójico) perturbar fuertemente el sistema para que puedan entrar en actividad los ciclos que dan resiliencia a los estados del ecosistema que se desean fomentar.

Propuesta de Investigación: Recuperación de áreas degradadas con un enfoque ecológico en Páramos del Volcán Chiles

En la actualidad, el mayor impacto sobre los páramos andinos es generado por la agricultura, la ganadería y las quemas asociadas a éstas cuya acción continuada causa pérdida gradual de coberturas vegetales (arbustivas y frailejonales), pérdida de suelo y transformación del paisaje. Por tanto, tomando como referencia esta problemática, se presenta a continuación un ejercicio académico donde se tratará de explicar la metodología a seguir para recuperar áreas degradadas en zonas paramunas del Volcán Chiles (localizadas en el Municipio de Cumbal, Departamento de
Restauración ecológica en los páramos

Nariño, entre los 0o 43' y Io 33' de Latitud Norte y desde los $77^{\circ} 17^{\prime}$ hasta los $77^{\circ} 57^{\prime}$ al oeste de Greenwich), a través de diferentes actividades de restauración ecológica, soportada con bases teóricas de ecología de la restauración (Figura 1):

1) A partir de investigaciones y experiencias anteriores en otras zonas y del enfoque conceptual de InvestigaciónAcción-Participación, la selección de las estrategias de restauración serán concertadas con la comunidad, de acuerdo con la cosmovisión de los páramos y su conservación. Respecto a la escala para establecer los objetivos de la restauración, Ehrenfeld (2000) recomienda la ecosistémica, en donde el objetivo de la restauración es principalmente la recuperación de algunas funciones del ecosistema.

2) Teniendo en cuenta que el Potencial Productivo del Suelo (PPS) es un indicador representativo del estado de bienestar del suelo (Zúñiga et al. 2009), en las zonas de bosque, frailejonal-pastizal y pastizal, identificadas como perturbadas bien sea por quemas o ganadería se levantarán mapas de la reserva energética del suelo, los cuales permitirán determinar una distribución espacial del PPS.

3) Para la selección de las especies vegetales a emplear en la restauración, se elegirán semillas y propágulos de plantas consideradas como pioneras, pioneras intermedias $y$ especies procedentes de bosque maduro.

4) En las zonas seleccionadas, de acuerdo con conceptos científicos y concepciones desde la cosmovisión de la comunidad; se establecerán las parcelas experimentales con las diferentes estrategias de restauración (siembra de especies vegetales, lluvia de semillas, sucesión natural). Las respectivas repeticiones se realizarán en sitios con diferentes niveles de PPS o que según la comunidad sean suelos "buenos" o "malos".

5) Se realizará monitoreo y evaluación durante dos años. Entre las variables a evaluar se tendrá en cuenta: a) Suelo: cambios en PPS, Materia orgánica, Nitrógeno, Fósforo, Potasio y pH. b) Cobertura Vegetal: Número de plantas ya establecidas, número de plantas nuevas, número de plantas que rebrotan, tiempo a la primera planta, tiempo al primer rebrote, Número de plantas y especies nativas que se establecen, Número de plantas y especies invasoras que se establecen, Riqueza de especies, Frecuencia; y c) Clima: intensidad lumínica, temperatura, humedad relativa, vientos y pluviosidad.

6) A partir de los resultados y datos obtenidos, se definirá el siguiente Índice: 
índice de Resiliencia $=$ índice de PPS x índice de la especie $\mathrm{x}$ índice del Clima

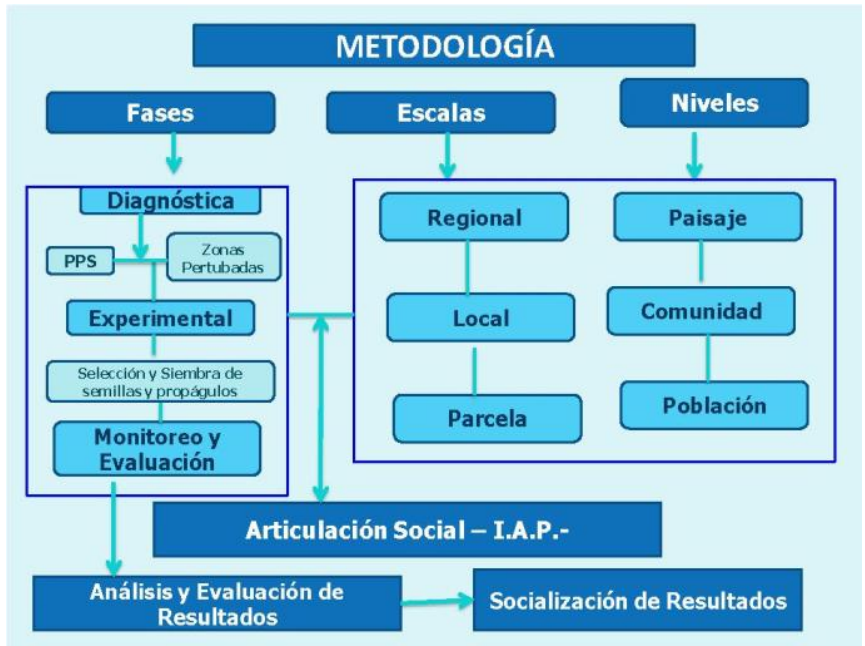

Figura 1. Esquema de la propuesta metodológica a desarrollar en la investigación.

Al determinar el índice de Resiliencia a partir de los parámetros propuestos se espera poder determinar cuáles son los sitios, especies y condiciones de clima que favorecen en un proceso de restauración ecológica para que el ecosistema se regenere más rápidamente. De acuerdo con Vargas \& Mora (2007), en una visión ecosistémica lo que se debe retornar a su estado predisturbio son las condiciones ecológicas que garantizan la recuperación de la composición, estructura y función del ecosistema, integrando los procesos a gran escala con los de pequeña escala.

Hipotéticamente, las zonas restauradas favorecidas por la intervención y manipulación humana iniciarán un proceso de cambios que favorecerán el sistema ecológico, para que éste logre un estado deseado.

La teoría y la práctica de la restauración ecológica empiezan por considerar los factores que regulan el desarrollo de estos sistemas, incluidos los humanos, y cuál es la disponibilidad para su recuperación. La restauración ecológica utiliza las relaciones entre los componentes de los ecosistemas (agua, suelo, plantas, animales, bacterias) simultáneamente para que el ecosistema en conjunto inicie una trayectoria de recuperación durante la cual el propio ecosistema se va organizando en relación con los factores que lo regulan.
El resultado final será un ecosistema, un espacio físico y los procesos que relacionan sus componentes, que está en equilibrio dinámico con los factores que lo regulan y con los otros espacios del entorno, es decir que se automantiene. Es, por tanto, sostenible y no requiere gastos de mantenimiento. Es también un ecosistema que proporciona beneficios de todo tipo (alimentos, contribuye a retener carbono en el suelo, mejora la calidad de agua) al conjunto del territorio o a particulares según las regulaciones a que esté sometido y los objetivos que se propongan, y por lo tanto, un ecosistema sostenible.

\section{REFERENCIAS}

BERTALANFFY, V. L. 1976. Teoría General de Sistemas. Petrópolis Vozes, México. 310 pp.

EHRENFELD, J. G. 2000. Defining the Limits of Restoration: The Need for Realistic Goals. Ecological restoration. The Journal of the Society for Ecological Restoration 8(1): 2-9.

GARCÍA, R. 1994. Intedisciplinariedad y sistemas complejos. En: LEFF, E. (ed). Ciencias Sociales y Formación Ambiental. Editorial Gedisa, Barcelona, España. 321 pp.

HOBBS, R. \& HUENNECKE, L. 1992. Disturbance, Diversity and Invasion. Implications for conservation Conservation Biology 6: 324-337.

HOFSTEDE, R. SEGARRA, P. \& MENA, V. 2003. Los páramos del mundo. Proyecto Atlas Mundial de los Páramos. Global Peatland Initiative/NC-IUCN/EcoCiencia. Quito, 299 pp.

JACKSON, L. LOPOUKHINE, N \& HILLYARD, D. 1995. Ecological restoration: a definition and comments. Restoration Ecology 3: 71-75.

JIMÉNEZ, P. J. AGUIRRE, O. TREVIÑO, E. GARZA, E. MEDELLÍN, G. ALANIS, F. \& CANALES, E. 2002. Priorización: Grados de Riesgo y Daño en el Área y Vegetación. En: Curso de Restauración de Áreas Quemadas para ONG'S Conservacionistas. Fondo Mexicano para la Conservación de la Naturaleza. Agencia para el Desarrollo Internacional de Estados Unidos.

JØRGENSEN, S. E. \& FATH, B. D. 2004. Application of thermodynamic principles in ecology", Ecological Complexity I: 267-280.

PICKETT, S. T. A. \& WHITE, P. S. 1985. The Ecology of Natural Disturbance and Patch Dynamics. Academic Press, EE.UU. 472 pp. 
PREMAUER J. \& VARGAS, O. 2004. Patrones de diversidad en vegetación pastoreada y quemada en un páramo húmedo (parque Natural Chingaza, Colombia). Ecotrópicos. 18(2): 52-66.

RANGEL-CH, J.O 2000. La región paramuna y franja aledaña en Colombia. Colombia Diversidad Biótica III. La región de vida paramuna. Universidad Nacional de Colombia. Instituto de Ciencias Naturales. Bogotá.

RIVAS, G. BACA, A. E. CALDERÓN, J. NARVÁEZ, G. RENGIFO, J. MUÑOZ, D. \& TORRES, C. 2007. Estado del Arte de los páramos del Departamento de Nariño: Componentes Físico Biótico y Socioeconómico. Vicerrectoría de Investigaciones Universidad de Nariño y Corponariño. Pasto, Colombia. Informe final Convenio Corponariño y Universidad de Nariño.

SER, SOCIETY FOR ECOLOGICAL RESTORATION INTERNATIONAL, Grupo de trabajo sobre ciencia y políticas. 2004. Principios de SER Internacional sobre la restauración ecológica. www.ser.org y Tucson: Society for Ecological Restoration Internacional.

SUDING, K. N. GROSS, K. L. \& HOUSEMAN, G.R. 2004. Alternative states and positive feedbacks in restoration ecology. Trends in Ecology and Evolution 19(1): 46-53.

VAN GIGCH, J. P. 1987. El enfoque de sistemas: Teoría General de Sistemas aplicada. En: Teoría general de sistemas, Capítulo 2. Ed. Trillas. 2a . Edición, México.

VARGAS. O. \& MORA, F. 2007. La Restauración Ecológica, $\mathrm{Su}$ Contexto, Definiciones y Dimensiones. En: Estrategias para la Restauración Ecológica del Bosque Alto andino.

http://www.redcre.org/pdf/restauracion_ecologica_cogua _c1.pdf. Consultado el 20 de abril de 2010. 